\title{
Adenoid Hypertrophy in Adults- A Prospective Study
}

\author{
Ashwini Shastry ${ }^{1}$, Roopa Malali ${ }^{1}$, Lyra Joy ${ }^{1}$, Archana $\mathrm{P}^{1}$ and Borlingegowda Viswanatha ${ }^{2 *}$ \\ ${ }^{1}$ Post-graduate residents, Department of ENT, Bangalore Medical College and Research Institute, India \\ ${ }^{2}$ Professor \& Head of ENT Department, Bangalore Medical College and Research Institute, India
}

*Corresponding author: Borlingegowda Viswanatha, Professor \& Head of ENT Department, Bangalore Medical College and Research Institute, India

\begin{abstract}
Adenoid enlargement is uncommon in adults and because examination of nasopharynx by indirect posterior rhinoscopy is inadequate, many cases of enlarged adenoid in adults are misdiagnosed and accordingly maltreated. Though adenoid hypertrophy is rare in adults, all patients presenting with nasal obstruction should undergo diagnostic nasal endoscopic examination. In the presence of hypertrophied adenoids, though the other causes of nasal obstruction are treated, symptoms may not be relieved unless patient undergoes adenoidectomy. In the present study, 36 cases of adenoid hypertrophy in adult patient is presented along with the review of literature.
\end{abstract}

Keywords: Adenoid; hypertrophy; adults

\section{Introduction}

The submucosal lymphoid tissue at the junction of roof and posterior wall of nasopharynx are known as adenoids. These are predominantly B cell organs amongst which the B cell lymphocytes comprise $50-65 \%$ of all adenoids and approximately $40 \%$ are $\mathrm{T}$ cell lymphocytes $3 \%$ are mature plasma cells [1]. Adenoids shows physiological enlargement up to 6 years of age and then they regress gradually during puberty and almost disappear completely by 16 years of age[2]. The enlargement of adenoid is uncommon in adults and most cases of enlarged adenoid in adults are misdiagnosed and they are maltreated[3]. Cases of adenoid hypertrophy in adults could be due to compromised immunity, especially those patients receiving organ transplants and in patients who are infected with human immunodeficiency virus (HIV)[2]. Enlarged adenoids can obstruct the airway resulting in nasal obstruction of varying degree, mouth breathing, snoring, nasal discharge, and nasal intonation of voice. Hypertrophied adenoids can also result in varied otologic symptoms due to blockage of Eustachian tube[4,5].

\section{Objective}

This descriptive study was done to evaluate the signs and symptoms associated with adenoid hypertrophy in adults.

\section{Materials and Methods}

This was a 3 years prospective descriptive study of adult patients, all aged more than 18 years with symptoms of nasal obstruction.

Study design: Prospective descriptive study

\section{Inclusion Criteria}

a) Patients who are willing to give an informed written consent.

b) Patients with history of nasal obstruction with enlarged adenoids on investigations.

c) Age of the patient more than 18 years. 
d) Patients who are willing for a regular follow up.

\section{Exclusion criteria}

a) Patients who are not willing to give informed written consent

b) Patients who are not willing for a regular follow up

\section{Methods}

The present study was conducted in the Department of Otorhinolaryngology, Bangalore Medical College and Research Institute, between January 2014 to December 2018. All the patients fulfilling the inclusion/ exclusion criteria were enrolled in the study. Detailed medical history was taken. Relevant past and family history were also taken into consideration. Anterior rhinoscopy wad done to find out deviated nasal septum, septal spur, hypertrophied turbinate's, nasal polyp, foreign body. Posterior rhinoscopy was done to examine the nasopharynx. The diagnosis of adenoid hypertrophy was made on the basis of the medical history, X-ray nasopharynx soft tissue lateral view, and endoscopy was obtained in an erect position with neck extended to visualize the shadow of the adenoid.Radiological assessment and grading of the size of adenoid was done accordingly [6]

a) Grade I: Soft tissue mass obstructing less than $25 \%$ of the nasopharyngeal airway.

b) Grade II: Soft tissue mass obstructing 25-50\% of the nasopharyngeal airway.

c) Grade III: Soft tissue mass obstructing 50- $75 \%$ of the nasopharyngeal airway.

d) Grade IV : Soft tissue mass obstructing more than $75 \%$ of the airway.

In endoscopic assessment, the size of the adenoid was determined according to Clemen's et al. grading[6]

a. Grade I:Adenoid tissue filling $1 / 3^{\text {rd }}$ of the vertical height of the choanae.

b. Grade II: Filling 2/3rd of the vertical height of the choanae.

c. Grade III: 2/3rd to nearly completely filling the choanae.

d. Grade IV: Completely filling the choanae.

After confirmation of the mass, adenoidectomy was performed and then the mass was sent for histopathological examination. The other causes of nasal obstruction if found on clinical assessment, such as deviated nasal septum were also treated simultaneously.

\section{Results}

The study group consisted of 36 cases. The ages ranged from 20- 40 years. 25 patients were between 20- 30 years of age and 11 patients belonged to $30-40$ years (Table 1 ). Out of the 36 cases,
20 patients were males and 16 patients were female (Table 2\& Figure1). Patients with adenoid hypertrophy were in 3rd decade of life and in our study we found that males were commonly affected than females in the ratio of 5:1.The presenting symptom was nasal obstruction in 34 patients, snoring was the main complaint in about 2 cases(Figures 2-4). 22 patients were diagnosed with adenoid hypertrophy during diagnostic nasal endoscopy for nasal obstruction, 14 patients were diagnosed with the adenoid hypertrophy with deviated nasal septum.Out of the 36 patients, 24 patients had Grade IV adenoid hypertrophy, 2 patients had grade 2 adenoid hypertrophy and in the remaining 10 patients adenoid tissue was found to fill more than $2 / 3$ rd of the vertical height of the choanae (Table 3\& Figure5).

Table 1: Showing age distribution.

\begin{tabular}{|c|c|}
\hline Age Group (Years) & Number of Patients \\
\hline $20-30$ & 25 \\
\hline $30-40$ & 11 \\
\hline
\end{tabular}

Table 2: Showing gender distribution.

\begin{tabular}{|c|c|}
\hline Gender Distribution & Number of Patients \\
\hline Male & 20 \\
\hline Female & 16 \\
\hline
\end{tabular}

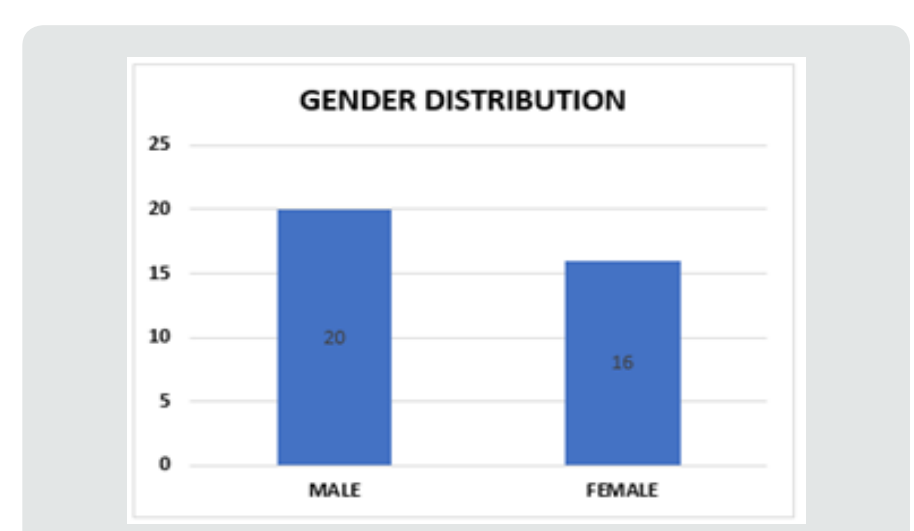

Figure 1: Showing gender distribution.

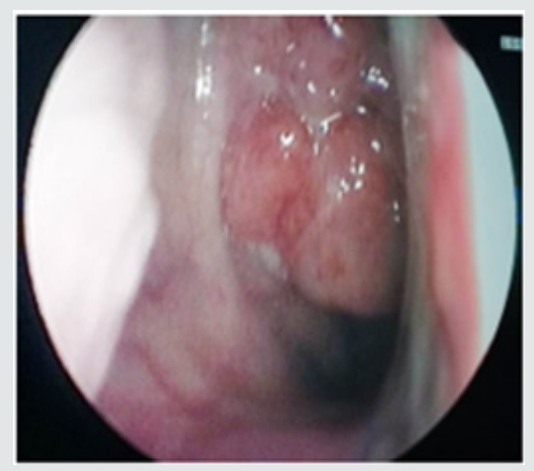

Figure 2: Nasal endoscopic picture showing Grade IIadenoid hypertrophy [filling $2 / 3$ rd of the vertical height of the choanae]. 


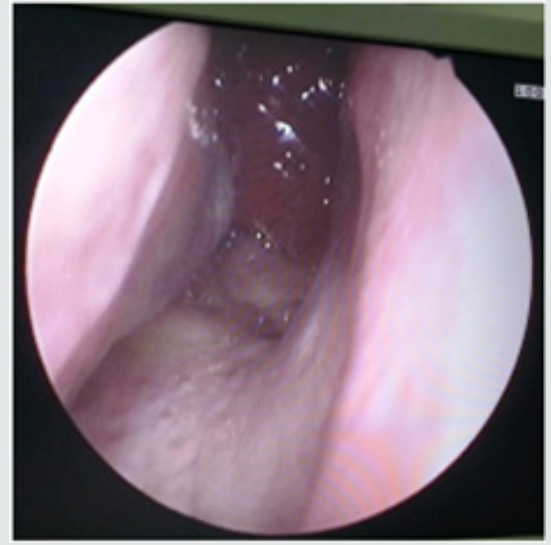

Figure 3: Nasal endoscopic picture showing Grade IIIadenoid hypertrophy [ $2 / 3$ rd to nearly completely filling the choanae].

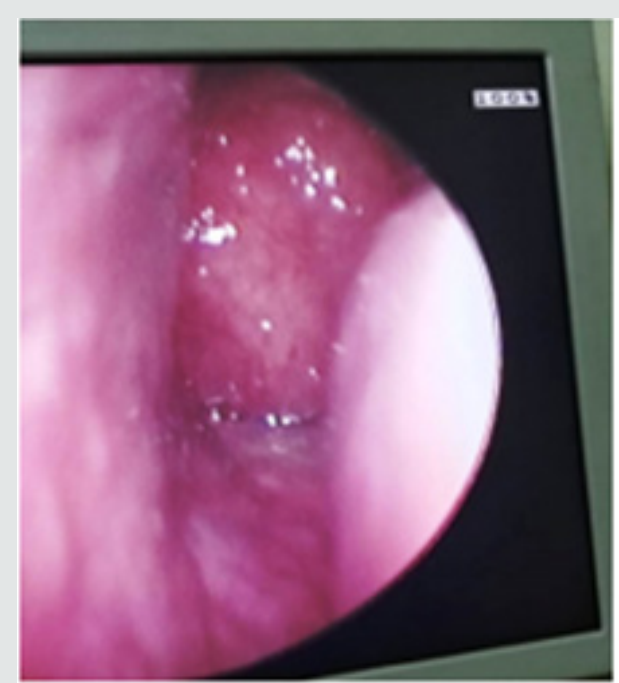

Figure 4: Nasal endoscopic picture showing Grade IVadenoid hypertrophy [completely filling the choanae].

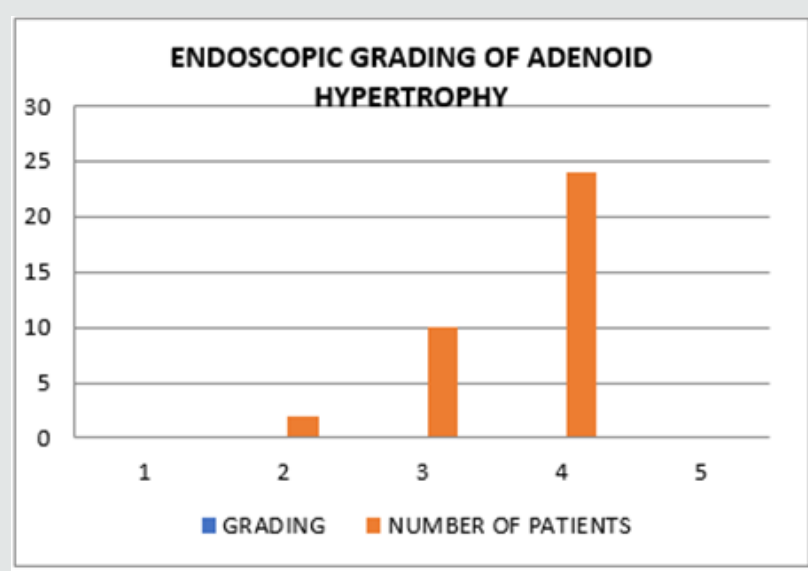

Figure 5: Showing endoscopic grading distribution.
Table 3: Showing endoscopic grading distribution.

\begin{tabular}{|c|c|}
\hline Grading & Number of Patients \\
\hline Grade 1 & 0 \\
\hline Grade II & 2 \\
\hline Grade III & 10 \\
\hline Grade IV & 24 \\
\hline
\end{tabular}

\section{Discussion}

The submucosal lymphoid tissue at the junction of roof and posterior wall of nasopharynx are called adenoids[6]. The nasopharyngeal lymphoid aggregate or Lushka's tonsil was described by Santorini in 1724.Nasopharyngeal vegetations were then described by Wilhem as "Adenoids" in 1870. Adenoids along with palatine tonsil, lingual tonsils, tubal tonsils, and lateral pharyngeal bands forms the inner Waldeyer's ring[6]. These are predominantly B cell organs which comprises of $50-65 \%$ of all adenoid lymphocyte and $40 \%$ are T cell lymphocytes and about $3 \%$ are mature plasma cells[1]. During the age of 3 and 10 years it shows greatest immunological activity and hence are most prominent during this period of childhood, and later shows involution[2].All the components of the Waldeyer's ring play a very important role in the immunological memory. The epithelium contains a system of specialized channels lined by M cells that take up antigens into the vesicles and transport them to the intra- and subepithelial spaces. Here they are presented to lymphoid cells. After passing through the epithelium, inhaled or ingested antigens reach the extrafollicular region or the lymphoid follicles. In the extrafollicular region, interdigitating cells (IDC) and macrophages process the antigens and present them to CD4+ T lymphocytes. Helper T cells then stimulate the proliferation of follicular B lymphocytes and their development into either antibody-expressing B memory cells capable of migration to the nasopharynx and other sites, or plasma cells that produce antibodies, and release them. The contact of memory B cells in the lymphoid follicles with antigen is an essential part of the generation of a secondary immune response.

Among the Ig isotypes, IgA may be considered the most important product of the adeno tonsillar immune system. In its dimeric form, IgA can attach to the transmembrane secretory component (SC) to form secretory IgA (SIgA), which is a critical component of the mucosal immune system of the upper airway. This component is necessary for the binding of IgA monomers to each other and to the SC and is an important product of B cell activity in the tonsil follicles. While the tonsils produce immunocytes bearing the J (joining) chain carbohydrate, the SC is produced only in the adenoid and extra tonsillar epithelium, and therefore, only the adenoid possesses a local secretory immune system[6].Adenoid enlargement is uncommon in adults and many cases of adenoid hypertrophy in adults are misdiagnosed[3]. Involuted adenoidal tissue may re-proliferate in response to infections and irritants 
resulting in adenoid hypertrophy. Adenoid hypertrophy in adults may occur in immunocompromised state, especially in those patients receiving organ transplants and also in those patients infected with having human immunodeficiency virus[5].

\section{Conclusion}

Though adenoid hypertrophy is rare in adults, all patients presenting with nasal obstruction should undergo diagnostic nasal endoscopic examination. In the presence of hypertrophied adenoids, even though the other causes of nasal obstruction are treated, symptoms may not be relieved unless patient undergoes adenoidectomy.

\section{References}

1. Cummings W, Charles S (2010) Textbook of Otolaryngology. $5^{\text {th }}$ Ed, 3(181): 2782-2784.
2. Yildirim N, Şahan M, Karslioğlu Y (2008) Adenoid hypertrophy in adults: clinical and morphological characteristics. Journal of International Medical Research 36(1): 157-162.

3. Kamel RH, Ishak EA (1990) Enlarged adenoid and adenoidectomy in adults: endoscopic approach and histopathological study. The Journal of Laryngology \& Otology 104(12): 965-967.

4. Sarma N, Khaund G (2017) A Comparative Study of Radiograph and Nasal Endoscopy in Diagnosis of Hypertrophied Adenoids. Indian Journal of Otolaryngology and Head \& Neck Surgery 71(3): 1793-1795.

5. Rout MR, Mohanty D, Vijaylaxmi Y, Bobba K, Metta C (2013) Adenoid hypertrophy in adults: a case series. Indian Journal of Otolaryngology and Head \& Neck Surgery 65(3): 269-274.

6. Darrow D, Derkay C, Mitchell R (2001) Bluestone, and stool's Pediatric otolaryngology. $5^{\text {th }}$ Ed, p.1192. 